\title{
Low-frequency index of refraction for a two-dimensional metallodielectric photonic crystal
}

\author{
A. A. Krokhin,,${ }^{1,2}$ E. Reyes, ${ }^{2}$ and L. Gumen ${ }^{3}$ \\ ${ }^{1}$ Department of Physics, University of North Texas, P.O. Box 311427, Denton, Texas 76203, USA \\ ${ }^{2}$ Instituto de Física, Universidad Autónoma de Puebla, Apartado Postal J-48, Puebla, 72570, Mexico \\ ${ }^{3}$ Universidad Popular Autonoma del Estado de Puebla, 21 Sur, \#1103, 72160, Mexico
}

(Received 27 September 2006; revised manuscript received 4 December 2006; published 29 January 2007)

\begin{abstract}
We calculate analytically the effective index of refraction $n_{\text {eff }}$ of a periodic arrangement of nonmagnetic metallic cylinders in the low-frequency limit. At $\omega \rightarrow 0$ the dielectric constant of the cylinders is singular, $\epsilon_{m}(\omega) \approx-\left(\omega_{p} / \omega\right)^{2}$, allowing propagation in the plane of periodicity of a mode with the magnetic field parallel to the cylinders ( $H$ polarization). The in-plane electric field induces eddy currents, which are localized in a narrow skin layer. We show that the magnetic moment of the eddy currents leads to diamagnetic response if the radius of the cylinders is larger than the skin depth $\delta \sim 10^{-5} \mathrm{~cm}$. Otherwise, the cylinders are transparent for the electromagnetic field and their magnetic moment can be neglected. Magnetization of the cylinders gives rise to distinct values of the quasistatic and static indices of refraction and explains a paradox with noncommuting limits $\epsilon_{m} \rightarrow \infty$ and $\omega \rightarrow 0$.
\end{abstract}

DOI: 10.1103/PhysRevB.75.045131

PACS number(s): 42.70.Qs, 41.20.Jb, 78.67.-n, 75.75.+a

Photonic crystals with metallic inclusions provide an example of an artificial medium possessing flat bands, ${ }^{1}$ wide band gaps, ${ }^{2}$ very low plasma frequency, ${ }^{3}$ and negative index of refraction. ${ }^{4}$ All these features are due to the large negative values of the dielectric constant of the metal, $\epsilon_{m}(\omega)=1$ $-\left(\omega_{p} / \omega\right)^{2}$, at frequencies well below the plasma frequency $\omega_{p}$. The highly conducting metallic inclusions shield their interiors from the electromagnetic field and the only region where the field penetrates is a narrow skin layer of width $\delta(\omega)=c /\left[\omega \sqrt{\left|\epsilon_{m}(\omega)\right|}\right]$. At low frequencies $\omega \ll \omega_{p}$, the skin depth is $\sim 10^{-5} \mathrm{~cm}$ for typical metals. If the size of the metallic inclusions greatly exceeds the skin depth, the narrow skin layer can be neglected and the interior of the metal can be considered as a perfect conductor with infinite dielectric permittivity. In this case the details of the field distribution inside the metal are lost but this has little effect on the dielectric properties of the metallodielectric composite. Since $\left|\epsilon_{m}(\omega)\right| \rightarrow \infty$ in the low-frequency limit, some effective parameters of the medium turn out to be independent of the properties of the metal, in particular of $\omega_{p}$. For example, the effective plasma frequency for the $E$-polarized mode and the effective dielectric constant for the $H$-polarized mode are determined only by the geometry of the unit cell.,5 This approximation of a perfect conductor is valid for good metals at low frequencies when dissipation is negligible.

The magnetic properties of the inclusions (which are supposed to be fabricated from a nonmagnetic metal) are due to the eddy currents induced by the propagating wave. ${ }^{6}$ The currents in the skin layer produce a magnetic moment, which depends on the geometry of the inclusions, the conductivity of the metal (i.e., the plasma frequency $\omega_{p}$ ), and the frequency of the propagating wave. ${ }^{7}$ Thus, the magnetic properties have to be calculated for each type of inclusion. It is also clear that the effect of magnetization on the electrodynamics of the medium is small if $\left|\epsilon_{m}(\omega)\right| \sim 1$ since the eddy currents are not localized and do not screen the interior of the inclusions from the electromagnetic wave. In this case the effective permeability of the medium $\mu_{e f f} \approx 1$. If, however, $\left|\epsilon_{m}(\omega)\right| \gg 1$ that happens at low frequencies $\omega \ll \omega_{p}$, magne- tization of the inclusions leads to a noticeable diamagnetic response. ${ }^{8}$ The diamagnetic response in the infrared and visible region of frequencies has been calculated recently in Ref. 9, using the coherent potential approximation. Our results obtained in the quasistatic limit are in agreement with the results of Ref. 9 .

Since the eddy currents always lead to a diamagnetic response, the quasistatic effective index of refraction $\sqrt{\epsilon_{e f f}} \mu_{e f f}$ is always less than the static index of refraction $\sqrt{\epsilon_{\text {eff }}}$. For metallic inclusions this difference may be very large and for special geometry of the inclusions, e.g., for split-ring resonators, the effective index of refraction may even become negative. ${ }^{4}$ It is much less obvious that the static and quasistatic indices of refraction are different for photonic crystals with dielectric inclusions. Here the surface currents are associated with the time-dependent vector of polarization $\dot{\mathbf{P}}$. For inclusions with $\epsilon \simeq 1$ the magnetic moment produced by the polarization currents gives a negligible contribution; therefore the static and quasistatic indices are practically equal. However, for inclusions with $\epsilon \gg 1$ the alternating polarization vector $\mathbf{P}(t)$ induces a magnetic moment, which may be comparable with that induced in a good conductor. Thus, the magnetic response has to be taken into account in the case of dielectric inclusions if $\epsilon \gg 1$.

The difference between the static and quasistatic indices of refraction was first mentioned in Ref. 10 where the band structure of dielectric cylinders with $\epsilon \gg 1$ arranged in a square lattice has been calculated numerically. The effective index of refraction of the periodic structure was extracted from the measurement of the slope of the acoustic band and compared with the static results for $\sqrt{\epsilon_{e f f}}$ obtained in Ref. 17. It was suggested that this difference is due to singular behavior of the wave equation for a medium with perfectly conducting cylinders, which is manifested in noncommutativity of the limits $\epsilon_{m} \rightarrow \infty$ and $\omega \rightarrow 0$. In a series of publications following Ref. 10 the idea of the noncommutativity of the limits has been elaborated and extended. ${ }^{11}$ A thorough mathematical analysis of the Helmholtz equation in a heterogeneous medium led to the conclusion that the singular behav- 
ior of the index of refraction originates from a large gradient of the electromagnetic field inside the inclusions with $\epsilon \gg 1$. Here we show that the physical reason for the difference between the static and quasistatic indices of refraction is the magnetization produced by the eddy currents. We obtain an analytical result for the effective index of refraction, which demonstrates that the metallic inclusions may exhibit metallic or dielectric behavior depending on the relation between the skin depth and the size of the inclusions. When the radius $a$ of the cylindrical inclusions gradually increases from zero, a transition from dielectric to metallic behavior occurs. Since this transition occurs in a narrow region of radii $a$, a sharp (but analytical) change in the index of refraction may be interpreted as a singularity. In our approach we do not meet the problem of noncommutativity of the limits, and propose a clear physical explanation of the difference between the static and quasistatic results for the effective index of refraction.

Examples of cases when the magnetic moment produced by the eddy currents becomes essential are well known in classical electrodynamics. We refer here to the problem of Rayleigh scattering at a dielectric sphere of radius $a$. For finite values of the dielectric constant $\epsilon$ the scattering cross section is given by ${ }^{12}$

$$
\sigma_{d}(\epsilon)=\frac{8 \pi}{3} \frac{\omega^{4} a^{6}}{c^{4}}\left|\frac{\epsilon-1}{\epsilon+2}\right|^{2} .
$$

In order to obtain the scattering cross section for a perfectly conducting sphere it is not enough to take the limit $\epsilon \rightarrow \infty$. It is also necessary to add the contribution from the magnetization produced by the eddy currents, which in this case is $25 \%$ of the scattering cross section (1) at a dielectric sphere, ${ }^{12}$

$$
\sigma_{m}=1.25 \sigma_{d}(\epsilon=\infty)=\frac{10 \pi}{3} \frac{\omega^{4} a^{6}}{c^{4}} .
$$

The coefficient 1.25 in Eq. (2) originates from metallic behavior of the dielectric sphere with $\epsilon=\infty$. A smooth analytical transition from Eq. (1) (dielectric behavior) to Eq. (2) (perfect-conductor behavior) can be obtained. It is given by the equation ${ }^{13}$

$$
\sigma(\epsilon)=\frac{8 \pi}{3} \frac{\omega^{4} a^{6}}{c^{4}}\left|\frac{\epsilon-1}{\epsilon+2}\right|^{2}\left(1+|\gamma|^{2}\right),
$$

which is valid for arbitrary $\epsilon$. Here the second term with

$$
\gamma=\frac{1}{2}\left(1+3 \frac{\cot q}{q}-\frac{3}{q^{2}}\right), \quad q=\omega a \sqrt{\epsilon} / c,
$$

describes the contribution of a magnetized dielectric sphere to the scattering cross section. Its value depends on the dimensionless parameter $q$, which is the ratio of the radius of the sphere to the wavelength in the dielectric. If the dielectric constant $\epsilon \sim 1$, this ratio is small in the Rayleigh approximation, $a \ll c / \omega$. Taylor expansion of Eq. (4) over the small parameter $q$ gives $\gamma \approx 0$. In the metallic limit, when not only $\epsilon \gg 1$ but also $q \gg 1$, the parameter $\gamma$ approaches $1 / 2$. The transition from dielectric to metallic behavior occurs at $q$ $\sim 1$, i.e., when the wavelength in the material becomes of the order of the size of the scatterer. The two different results obtained from Eq. (4) in the limits $\epsilon \rightarrow \infty(q \rightarrow \infty)$ and $\omega$ $\rightarrow 0(q \rightarrow 0)$ may be formally interpreted as noncommutativity of the limits. For inclusions with temporal dispersion $\left|\epsilon_{m}(\omega)\right|=\left(\omega_{p} / \omega\right)^{p}$ the parameter $q$ approaches zero (dielectric behavior) for $p>2$ in the long-wavelength limit, and approaches infinity for $p<2$ (metallic behavior), independently of the size of the inclusions. This conclusion is in agreement with the results obtained in Ref. 11. The case of the free electron plasma, $p=2$, is the only case when the parameter $q$ remains finite in the long-wavelength limit. Here it also depends on the plasma frequency $\omega_{p}$ and the radius $a$ of the inclusions, giving the possibility for realization of dielectric $\left(q=\omega_{p} a / c \ll 1\right)$ and metallic $\left(q=\omega_{p} a / c \gg 1\right)$ behavior.

A very similar physical situation appears in the theory of homogenization of metallodielectric photonic crystals. The wave equation for the $H$ mode is written as follows:

$$
\boldsymbol{\nabla} \cdot\left(\frac{1}{\varepsilon(\mathbf{r})} \boldsymbol{\nabla} H\right)=\frac{\omega^{2}}{c^{2}} \mu(\mathbf{r}) H
$$

Since we consider the case of nonmagnetic constituents, values of $\mu(\mathbf{r})$ different from 1 may appear only due to the magnetic moment $M=\pi a^{2} \alpha H$ acquired by a cylinder of radius $a$. For the magnetic field parallel to the axis the magnetization per unit length is given by ${ }^{13}$

$$
\alpha=-\frac{1}{4 \pi}\left(1-\frac{2}{k a} \frac{J_{1}(k a)}{J_{0}(k a)}\right) .
$$

Here $k=(\omega / c) \sqrt{\bar{\epsilon}}$ is the complex wave number in the cylinder and $J_{0}(x)$ and $J_{1}(x)$ are the Bessel functions. For the case of a collisionless plasma at low frequencies $\omega \ll \omega_{p}$, the parameter $k a$ is pure imaginary, $k a \approx i a / \delta_{0}$, where $\delta_{0}=c / \omega_{p}$ is the limiting value of the skin depth. A metallic cylinder with currents circulating in the skin layer can be considered as a diamagnetic cylinder with magnetic permeability

$$
\mu_{m}=\frac{2 \delta_{0}}{a} \frac{I_{1}\left(a / \delta_{0}\right)}{I_{0}\left(a / \delta_{0}\right)} .
$$

Here $I_{0}(x)$ and $I_{1}(x)$ are the modified Bessel functions of the first kind. This low-frequency permeability is independent of $\omega$ but it depends on the plasma frequency $\omega_{p}$ and cylinder radius $a$. As for a dielectric sphere there are two distinct physical situations. For very thin cylinders, $a \ll \delta_{0}$, the metallic inclusions are practically transparent for radiation and their magnetic properties can be neglected. Expansion of the right-hand side of Eq. (7) gives $\mu_{m} \approx 1-\left(a / 2 \delta_{0}\right)^{2}$. This case is realized for metallic cylinders with radius $a \leq 10^{-5} \mathrm{~cm}$. Thicker cylinders exhibit diamagnetic behavior, i.e., $\mu<1$. If $a \gg \delta_{0}$ Eq. (7) gives a close to zero value of the permeability $\mu_{m} \approx 2 \delta_{0} / a \ll 1$, i.e., the inclusions can be considered as ideal diamagnetic cylinders.

The low-frequency index of refraction is defined as 


$$
n_{e f f}(\hat{\mathbf{k}})=\lim _{k \rightarrow 0} \frac{c k}{\omega}
$$

This limit depends on the direction of propagation $\hat{\mathbf{k}}=\mathbf{k} / k$ and it is obtained from the wave equation (5) where $\epsilon(\mathbf{r})$ and $\mu(\mathbf{r})$ are periodic functions in the plane perpendicular to the cylinders $(x-y$ plane $)$. To calculate the limit (8) we apply the method of homogenization developed in Refs. 5 and 14-16. The plane-wave expansion of Eq. (5) leads to the following set of linear equations for the Fourier components of the Bloch wave $H(\mathbf{r})=\exp (i \mathbf{k} \cdot \mathbf{r}) \Sigma_{\mathbf{G}} h_{k}(\mathbf{G}) \exp (i \mathbf{G} \cdot \mathbf{r})$ :

$$
\begin{gathered}
\sum_{\mathbf{G}^{\prime}} \eta\left(\mathbf{G}-\mathbf{G}^{\prime}\right)(\mathbf{k}+\mathbf{G}) \cdot\left(\mathbf{k}+\mathbf{G}^{\prime}\right) h_{k}\left(\mathbf{G}^{\prime}\right) \\
=\left(\omega^{2} / c^{2}\right) \sum_{\mathbf{G}^{\prime}} \mu\left(\mathbf{G}-\mathbf{G}^{\prime}\right) h_{k}\left(\mathbf{G}^{\prime}\right) .
\end{gathered}
$$

Here $\mathbf{G}$ are the reciprocal-lattice vectors. The Fourier coefficients of the periodic functions $\eta(\mathbf{r})=\epsilon^{-1}(\mathbf{r})$ and $\mu(\mathbf{r})$ are determined as follows:

$$
\begin{gathered}
\eta(\mathbf{G} \neq \mathbf{0})=\frac{1}{A_{c}} \int_{c y l}\left(\frac{1}{\epsilon_{m}(\omega)}-\frac{1}{\epsilon_{b}}\right) \exp (-i \mathbf{G} \cdot \mathbf{r}) d \mathbf{r}, \\
\mu(\mathbf{G} \neq \mathbf{0})=\frac{1}{A_{c}} \int_{c y l}\left(\mu_{m}(\omega)-1\right) \exp (-i \mathbf{G} \cdot \mathbf{r}) d \mathbf{r}
\end{gathered}
$$

Here $A_{c}$ is the area of the unit cell, the integrals are taken over the cross section of the cylinder, and $\epsilon_{b}$ is the dielectric constant of the background material. The Fourier components with $\mathbf{G}=\mathbf{0}$ are reduced to the bulk average values,

$$
\begin{gathered}
\eta(G=0) \equiv \bar{\eta}=\frac{f}{\epsilon_{m}(\omega)}+\frac{1-f}{\epsilon_{b}}, \\
\mu(G=0) \equiv \bar{\mu}=f \mu_{m}(\omega)+1-f,
\end{gathered}
$$

where $f$ is the filling fraction of the metallic component and $\epsilon_{b}$ is the dielectric constant of the background material.

In the low-frequency limit the Fourier components $\eta(\mathbf{G})$ and $\bar{\eta}$ become independent of the frequency $\omega$ and the dielectric constant of the metal $\epsilon_{m}$,

$$
\eta(\mathbf{G} \neq \mathbf{0}) \approx-\frac{1}{A_{c} \boldsymbol{\epsilon}_{b}} \int_{c y l} \exp (-i \mathbf{G} \cdot \mathbf{r}) d \mathbf{r} .
$$

With the same accuracy the magnetic permeability of the cylinders in Eqs. (11) and (13) can by substituted by the $\omega$-independent value given by Eq. (7). Thus Eq. (9) is reduced to the generalized eigenvalue problem for a magnetodielectric photonic crystal. A homogenization procedure for this problem has been proposed in Ref. 16 and the effective index of refraction has been calculated. Due to the continuity of the magnetic field at the surface of the cylinders the effective permeability coincides with the bulk average value $\bar{\mu}$ given by Eq. (13). Then for the index of refraction for the $H$-polarized mode one obtains

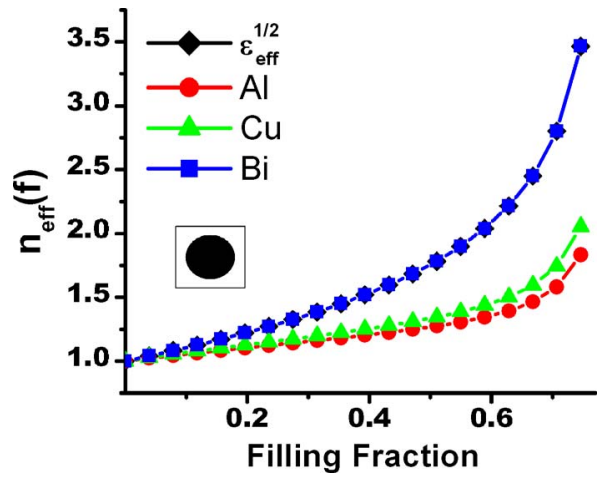

FIG. 1. (Color online) The effective index of refraction $n_{\text {eff }}$ $=\sqrt{\mu_{e f f} \varepsilon_{e f f}}$ for the $H$ mode for a photonic crystal of metallic cylinders arranged in a square lattice. The cylinders are of three different metals: aluminum (circles), copper (triangles), and bismuth (squares). We also plot the static effective index of refraction $\sqrt{\varepsilon_{\text {eff }}}$, which almost coincides with the effective index of refraction of bismuth cylinders.

$$
n_{e f f}^{2}=\bar{\mu} \epsilon_{e f f}(\hat{\mathbf{k}})=\left(1-f+\frac{2 f \delta_{0}}{a} \frac{I_{1}\left(a / \delta_{0}\right)}{I_{0}\left(a / \delta_{0}\right)}\right) \epsilon_{e f f}(\hat{\mathbf{k}})
$$

where the effective dielectric constant is given by ${ }^{14,15}$

$$
\begin{aligned}
\boldsymbol{\epsilon}_{e f f}(\hat{\mathbf{k}})= & \left\{\bar{\eta}-\sum_{\mathbf{G}, \mathbf{G}^{\prime} \neq 0}(\hat{\mathbf{k}} \cdot \mathbf{G})\left(\hat{\mathbf{k}} \cdot \mathbf{G}^{\prime}\right) \eta(\mathbf{G}) \eta\left(-\mathbf{G}^{\prime}\right)\right. \\
& \left.\times\left[\mathbf{G} \cdot \mathbf{G}^{\prime} \eta\left(\mathbf{G}-\mathbf{G}^{\prime}\right)\right]^{-1}\right\}^{-1} .
\end{aligned}
$$

Here $[\cdots]^{-1}$ stands for the matrix inversion in $\mathbf{G}$ space. Equations (15) and (16) are exact and valid for any geometry of the unit cell and arbitrary filling fraction. The dependence on the direction of propagation $\hat{\mathbf{k}}$ in Eq. (16) gives an ellipse in the $x-y$ plane. ${ }^{14,15}$ It becomes a circle if the crystal possesses a third- or higher-order rotational axis of symmetry. The obtained results are valid when the direction of propagation is in the plane of periodicity, $\hat{k_{z}}=0$. This is the only case when an electromagnetic wave propagates in the low-frequency limit. Any deviation from this plane induces infinite currents along the cylinders, i.e., the effective dielectric constant becomes infinite. At the same time the effective permeability remains finite and it can be calculated using the formulas given in Ref. 16.

The effective index of refraction Eq. (15) depends on the filling fraction. In Fig. 1 this dependence is plotted for the cases of metallic cylinders made of aluminum, copper, and bismuth and arranged in a square lattice with period $d=1.5$ $\times 10^{-4} \mathrm{~cm}$ in air, $\varepsilon_{b}=1$. The low-frequency permittivities $\epsilon_{e f f}$ of these metallic structures are universal (material independent) and they are calculated numerically, substituting $\epsilon_{m}$ $=\infty$ in Eq. (16)..$^{5}$ The dependence $\sqrt{\epsilon_{e f f}(f)}$ is shown in Fig. 1, where it practically coincides with the curve $n_{e f f}(f)$ obtained 

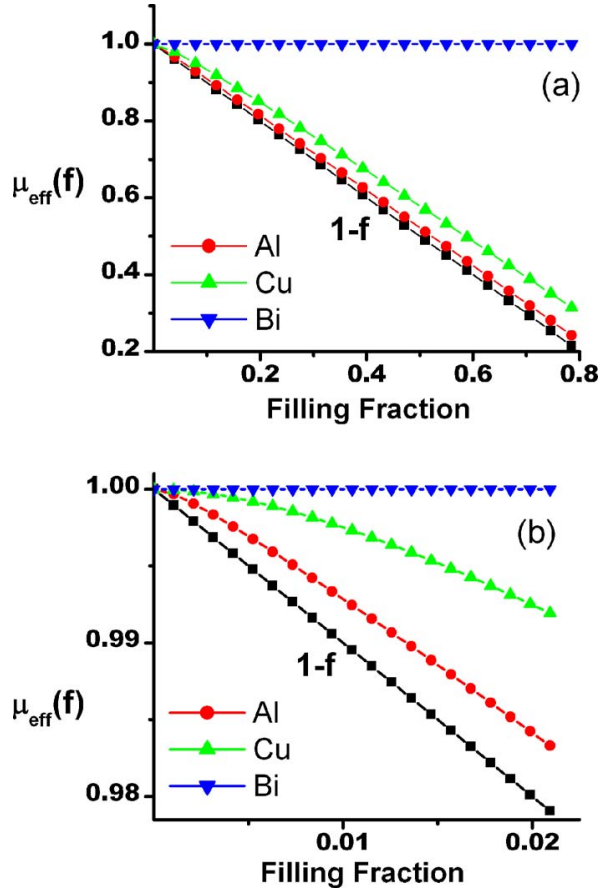

FIG. 2. (Color online) The effective permeability vs filling fraction for (a) the whole range of filling fractions and (b) the transition region of small filling fractions.

for the bismuth cylinders. Since bismuth is a semimetal with low plasma frequency, $\omega_{p}^{\mathrm{Bi}}=9.8447 \mathrm{THz}$, the corresponding skin depth $\delta_{0}^{\mathrm{Bi}}=c / \omega_{p}^{\mathrm{Bi}}=3.05 \times 10^{-3} \mathrm{~cm}$ exceeds the maximum possible cylinder radius $a_{\max }=d / 2=0.75 \times 10^{-4} \mathrm{~cm}$ at the close-packing filling fraction $f_{\max }=\pi / 4$. In this case, according to Eq. (7) the conducting bismuth cylinder does not exhibit diamagnetism, i.e., $n_{e f f}=\sqrt{\epsilon_{e f f}}$. In other words, the electric response of the bismuth cylinders is calculated in the perfect-metal approximation, $\left(\epsilon_{m}=\infty\right)$; however, their magnetic response (for radii $a \leq a_{\max }$ ) is negligible because of the relatively low concentration of conduction electrons. With respect to the magnetic component of the propagating wave the material of the cylinders behaves like a dielectric. The latter means that the effective index of refraction of a periodic structure of bismuth cylinders can be calculated using the pure electrostatic approach developed in Ref. 17. In fact, the numerical values of the effective dielectric constant $\epsilon_{e f f}$ in Fig. 1 coincide with those calculated in Ref. 10, using the electrostatic approach, in the whole range of the filling fraction.

The above result does not hold for the aluminum and copper photonic crystals. These metals are good conductors with plasma frequencies $\omega_{p}^{\mathrm{Al}}=22788.7 \mathrm{THz}$ and $\omega_{p}^{\mathrm{Cu}}$ $=6077.4 \mathrm{THz}$ and skin depths $\delta_{0}^{\mathrm{Al}}=1.32 \times 10^{-6} \mathrm{~cm}$ and $\delta_{0}^{\mathrm{Cu}}$ $=4.94 \times 10^{-6} \mathrm{~cm}$. Since in this case $\delta_{0} \ll a_{\max }$, the electromagnetic radiation does not penetrate inside the cylinders and they exhibit diamagnetic behavior practically for all values of $f$. In Fig. 2(a) we plot the effective permeability vs filling fraction for $\mathrm{Al}, \mathrm{Cu}$, and $\mathrm{Bi}$ photonic crystals. For the good conductors aluminum and cooper, the permeability (13) is close to the dependence $\mu_{\text {eff }}=1-f$, which is the permeability of a photonic crystal of ideally diamagnetic cylinders with $\mu_{m}=0$. In real metals dispersion and dissipation lead to saturation of the diamagnetic response. It is saturated at the level of $\mu_{e f f} \approx 0.8 .^{9}$ For bismuth there in no magnetic response, $\mu_{e f f}=1$. Because of the different magnetic behavior of the material cylinders, the curves for the effective index of refraction in Fig. 1 turn out to be different. It is clear that the magnetic response cannot be calculated in the framework of the electrostatic approach. Since the cylinders are nonmagnetic, their index of refraction formally may be attributed to the dielectric response only: $n_{\text {eff }}=\sqrt{\epsilon_{e f f}}$. In this approach developed in Refs. 10 and 11 it is assumed that $n_{e f f}=\sqrt{\epsilon_{e f f}}$ and the magnetic properties of the cylinders are not separated from their dielectric properties. The difference between the static and quasistatic indices of refraction is then explained by noncommutativity of the limits.

The diamagnetic response of the conducting cylinders gradually increases with the filling fraction since they become less and less transparent when the radius $a$ increases. The transition from the dielectriclike behavior with $\mu_{\text {eff }} \approx 1$ to the ideal-diamagnetic-like behavior with $\mu_{\text {eff }} \approx 1-f$ occurs in the region of very small filling fractions, $f \simeq 0.05$, where $a \simeq \delta_{0}$. This transition is shown in Fig. 2(b). Although the effective permeabilities for the good metals $\mathrm{Al}$ and $\mathrm{Cu}$ are close to the ideal-diamagnetic line, the distinction is still visible even for $\mathrm{Al}$. This is because the parameter $\delta_{0}^{\mathrm{Al}} / a$ is not sufficiently small. At the close-packing filling it is about 0.02 . The asymptotical behavior $n_{\text {eff }}=\sqrt{(1-f) \epsilon_{\text {eff }}}$ has been calculated by Poulton et al., ${ }^{11}$ Felbacq, ${ }^{18}$ and later $\mathrm{Hu}$ and Chan, ${ }^{19}$ using the Dirichlet boundary condition at the surface of the cylinders. This condition corresponds to the cylinders of an ideal diamagnetic. Our method of calculation of $n_{\text {eff }}$ based on the Fourier expansion does not use any boundary conditions at the surfaces of the cylinders.

The aforementioned difference between $\mu_{\text {eff }}$ and $1-f$ for periodic structures with inclusions of good metals is clear evidence that it is much more difficult to design a photonic crystal with negative $\mu_{\text {eff }}$ than one with negative $\epsilon_{\text {eff }}$. A particular spatial geometry of the inclusions is necessary in order to overcome the critical value of $\mu_{\text {eff }}=0$ in metamaterials fabricated from real metals where dissipation plays an important role. 4,6

In conclusion, we calculated the index of refraction for two-dimensional photonic crystals containing either metallic cylinders or dielectric cylinders with $\epsilon \ll 1$. It is shown that, due to the eddy currents (or polarization currents) induced by the low-frequency wave, a nonmagnetic photonic crystal exhibits a diamagnetic response. This response depends on the relation between the radius of the cylinder and the skin depth (wavelength inside the inclusion). If the skin depth is smaller than the radius of the cylinder, the interior of the inclusion is screened from the electromagnetic field. Each inclusion behaves like an ideal-diamagnetic cylinder with $\mu=0$. For the $H$-polarized wave the macroscopic magnetic permeability of the photonic crystal as a whole decreases linearly with increasing filling fraction, $\mu_{e f f}=1-f$. In the opposite case, when the skin layer is larger the radius, the diamagnetic 
response is negligibly small. Unlike the magnetic permeability, the effective dielectric permittivity turns out to be material independent and it is calculated assuming that the cylinders are perfect metals with $\epsilon=\infty$. We also explain the problem of the noncommuting limits, which appears if the index of refraction is attributed only to the dielectric response of the photonic crystal.

This work was supported by the DOE Grant No. DEFG02-06ER46312 and by CONACyT Grant No. 42136-F.
${ }^{1}$ A. R. McGurn and A. A. Maradudin, Phys. Rev. B 48, 17576 (1993).

${ }^{2}$ E. R. Brown and O. B. McMahon, Appl. Phys. Lett. 67, 2138 (1995); S. Fan, P. R. Villeneuve, and J. D. Joannopoulos, Phys. Rev. B 54, 11245 (1996); M. M. Sigalas, C. T. Chan, K. M. Ho, and C. M. Soukoulis, ibid. 52, 11744 (1995); A. Moroz, Phys. Rev. Lett. 83, 5274 (1999); J. G. Fleming, S. Y. Lin, I. El-Kady, R. Biswas, and K. M. Ho, Nature (London) 417, 52 (2002).

${ }^{3}$ J. B. Pendry, A. J. Holden, W. J. Stewart, and I. Youngs, Phys. Rev. Lett. 76, 4773 (1996).

${ }^{4}$ J. B. Pendry and D. R. Smith, Phys. Today 57 (6), 37 (2004).

${ }^{5}$ P. Halevi, A. A. Krokhin, and J. Arriaga, Phys. Rev. Lett. 86, 3211 (2001).

${ }^{6}$ V. M. Shalaev, W. Cai, U. K. Chettiar, H.-K. Yuan, A. K. Sarychev, V. P. Drachev, and A. V. Kildishev, Opt. Lett. 30, 3356 (2005); A. V. Kildishev, W. Cai, U. K. Chettiar, H.-K. Yuan, A. K. Sarychev, V. P. Drachev, and V. M. Shalaev, J. Opt. Soc. Am. B 23, 423 (2006).

${ }^{7}$ A. N. Lagarkov and A. K. Sarychev, Phys. Rev. B 53, 6318 (1996).

${ }^{8} \mathrm{We}$ assume that the inclusions do not form conducting clusters, unlike the situation considered in Ref. 7. Therefore paramagnetic response is absent

${ }^{9}$ X. Hu, C. T. Chan, J. Zi, M. Li, and K.-M. Ho, Phys. Rev. Lett.
96, 223901 (2006).

${ }^{10}$ N. A. Nicorovici, R. C. McPhedran, and L. C. Botten, Phys. Rev. Lett. 75, 1507 (1995).

${ }^{11}$ N. A. Nicorovici, R. C. McPhedran, and L. C. Botten, Phys. Rev. E 52, 1135 (1995); R. C. McPhedran, N. A. Nicorovici, and L. C. Botten, J. Electromagn. Waves Appl. 11, 981 (1997); C. G. Poulton, L. C. Botten, R. C. McPhedran, N. A. Nicorovici, and A. B. Movchan, SIAM J. Appl. Math. 61, 1706 (2001); C. Poulton, S. Guenneau, and A. B. Movchan, Phys. Rev. B 69, 195112 (2004).

${ }^{12}$ J. D. Jackson, Classical Electrodynamics, 3rd ed. (Wiley, New York, 1999).

${ }^{13}$ L. D. Landau, E. M. Lifshitz, and L. P. Pitaevskii, Electrodynamics of Continuous Media, 2nd ed. (Pergamon, Oxford, 1984).

${ }^{14}$ P. Halevi, A. A. Krokhin, and J. Arriaga, Phys. Rev. Lett. 82, 719 (1999).

${ }^{15}$ A. A. Krokhin, P. Halevi, and J. Arriaga, Phys. Rev. B 65, 115208 (2002).

${ }^{16}$ A. A. Krokhin, and E. Reyes, Phys. Rev. Lett. 93, 023904 (2004).

${ }^{17}$ W. T. Perrins, D. R. McKenzie, and R. C. McPhedran, Proc. R. Soc. London, Ser. A 369, 207 (1979).

${ }^{18}$ D. Felbacq, J. Math. Phys. 43, 52 (2002).

${ }^{19}$ X. Hu and C. T. Chan, Phys. Rev. Lett. 95, 154501 (2005). 